\title{
RELAÇÃO ENTRE O CONDICIONAMENTO FÍSICO E A IDADE NO DESEMPENHO DE EQUIPES DE CORRIDA DE AVENTURA
}

DOI: 105902/010283089794 Data de Submissão: 11/07/2013 Data de Aceite: 28/01/2014

Márcio de Avila Palermo Educador Físico, Universidade Federal do Rio Grande do Sul equipe@runningsports.com.br

Fernanda Dapper Coelho Educador Físico, Universidade Federal do Rio Grande do Sul fedapper@yahoo.com.br

Tatiane Gorski

Educador Físico, Universidade Federal do Rio Grande do Sul tatigorski@gmail.com

Marcelo Coertjens

Professor Assistente da Universidade Federal do Piauí coertjens@hotmail.com

Luiz Fernando Martins Kruel Professor Associado da Universidade

Federal do Rio Grande do Sul kruel@esef.ufrgs.br

RESUMO: O objetivo deste estudo foi correlacionar valores de consumo máximo de oxigênio $\left(\mathrm{VO}_{2 \text { max }}\right)$ e variáveis avaliadas no segundo limiar ventilatório $\left(2^{\circ} \mathrm{LV}\right)$ de equipes de Corrida de Aventura. Seis equipes realizaram teste progressivo em esteira para determinação do $\mathrm{VO}_{2 \operatorname{máx}}$ e do $2^{\circ} \mathrm{LV}$. Não foram encontradas diferenças significativas entre as médias das equipes e sua correlação com o desempenho foi baixa. Bons coeficientes foram encontrados entre idade e desempenho para navegadores $(r=-0,77)$ e mulheres $(r=-0,90 ; p<0,05)$. Apesar do condicionamento físico ser requisito ao esporte, a experiência dos navegadores e da mulher pode ter contribuído para o desempenho das equipes.

Palavras-Chave: Aptidão Física. Consumo de Oxigênio. Esportes. 


\section{Introdução}

A Corrida de Aventura (CA) é um esporte de caráter competitivo criado a partir da união de modalidades esportivas. As mais freqüentes são a Corrida de Orientação (CO), o Mountain Bike, técnicas da escalada e a Canoagem, podendo ser incluídas outras tais como a Vela, o mergulho, a Natação, a Cavalgada, etc. Apesar de existirem diferenças no que se refere às modalidades oferecidas e às regras das competições, encontram-se características comuns que sustentam um padrão de funcionamento. As competições são disputadas por equipes, normalmente quartetos mistos (no mínimo uma pessoa do sexo oposto), que se orientam por meio de bússolas e mapas da largada até a chegada sem percurso pré-definido, sendo obrigatória a passagem pelos pontos de controle (PC), vencendo a equipe completa que primeiro cruzar a linha de chegada tendo passado por todos os PCs.

O esporte teve origem em 1989 na Nova Zelândia, e no Brasil apareceu em 1998 com a Expedição Mata Atlântica (EMA). Apesar do amadorismo entre a maioria de seus praticantes, onde muitos são atletas das modalidades citadas, amantes e praticantes de esportes em meio à natureza, tem se observado uma regularidade no desempenho de algumas equipes em importantes competições. Este fato pode estar revelando uma dedicação e especificidade do treinamento destas equipes visando aprimorar as valências fisiológicas, psicológicas, táticas e técnicas importantes para o sucesso nestas competições.

A identificação das valências peculiares a cada esporte é de fundamental importância a fim de se buscar um treinamento específico e assim almejar sempre resultados mais significativos. Trabalhos mais recentes que buscaram identificar fatores determinantes no desempenho final de atletas em competições de triatlo, cross-country e maratonas, vêm defendendo o conceito da multifatoriedade. Este conceito ressalta a contribuição de diversos fatores fisiológicos, biomecânicos, psicológicos e técnicos atuando como determinantes no desempenho final de atletas em competições de longa duração (CAVANAGH; KRAM, 1985; DANIELS, 1985; NOAKES, 1988; MARTIN; MORGAN, 1992; CREAGH; REILLY, 1997; BASSETT; HOWLEY, 2000; NOAKES, 2000).

No que se refere aos fatores fisiológicos, a literatura tem apontado o consumo máximo de oxigênio $\left(\mathrm{VO}_{2 \text { máx. }}\right)$ e segundo limiar de lactato $\left(2^{\circ} \mathrm{LL}\right)$ como fatores importantes nos 
estudos de predição de desempenho esportivo. Um elevado $\mathrm{VO}_{2 \text { máx }}$, apesar de não ser considerado um fator propriamente dito de predição de desempenho entre atletas de elevado condicionamento físico, é visto como uma pré condição para estes alcançarem o sucesso em competições como o ciclismo, corridas de média e longa distância, natação e triatlo (KOHRT et al. 1987; BUTTS et al. 1991; SLEIVERT; ROWLANDS, 1996). Em relação ao $2^{\circ} \mathrm{LL}$, existe um consenso em relação a sua importância na determinação do desempenho esportivo (BASSETT; HOWLEY, 2000; NOAKES, 2000). No entanto, métodos alternativos são utilizados para indiretamente determinar o ponto de predominância do metabolismo anaeróbico e correlacioná-lo com a performance. Uma alternativa menos invasiva de detecção do $2^{\circ}$ LL e uma forma indireta de avaliação do metabolismo aeróbico e anaeróbico do atleta que pode ser obtida através da análise das respostas ventilatórias durante exercício (CAIOZZO et al. 1982) é realizar a determinação do segundo limar ventilatório (2LV) (DICKHUTH et al. 1999).

De uma forma geral, ao compararmos atletas com elevados valores de $\mathrm{VO}_{2 \text { máx }}$, aquele que apresentar um $2^{\circ} \mathrm{LV}$ mais próximo do seu $\mathrm{VO}_{2 \text { máx }}$ possuirá, provavelmente, melhores condições de performance do que um atleta com $2^{\circ} \mathrm{LV}$ distante. Isso possibilita ao atleta uma maior economia de movimento, visto que conseguiria correr em uma determinada intensidade com menor custo metabólico ou, então, sofrendo menor efeito da acidose (BASSETT; HOWLEY, 2000). Dentre os parâmetros utilizados para correlacionar o $2^{\circ} \mathrm{LV}$ com a performance podemos citar $\mathrm{O} \mathrm{VO}_{2}$ correspondente ao segundo limiar ventilatório $\left(\mathrm{VO}_{2} 2^{\circ} \mathrm{LV}\right)$ (TANAKA et al. 1984), o percentual (\%) que este se encontra em relação ao $\mathrm{VO}_{2 \text { máx }}\left(\% 2^{\circ} \mathrm{LV}\right)(P E ́ R O N N E T$ et al. 1987) e a intensidade (watts) ou a velocidade do exercício ( $\left.\mathrm{m}^{*} \mathrm{~min}-1\right)$ correspondente ao $2^{\circ} \mathrm{LV}($ Vel_2ㄴV) (TANAKA et al. 1984; MAFFULLI et al. 1991; SLEIVERT; WENGER, 1993).

Neste sentido, a CA é um esporte que pode, também, se enquadrar dentro do conceito de multifatoriedade, pois, somando-se às qualidades fisiológicas, existem as habilidades técnicas e cognitivas de seus participantes influenciando a performance. Em uma revisão bibliográfica, não foram encontradas pesquisas referentes aos fatores fisiológicos determinantes do desempenho em CA, entretanto, diversos autores, ao analisarem a CO, tem demonstrado que esse esporte possui predominância aeróbica (ACKERMAN et al. 
1985; SMEKAL et al. 2003) com importante contribuição do metabolismo anaeróbico em situações específicas ao longo da prova (KNOWLTON et al. 1980; DRESEL, 1985; AINSLIE et al. 2002). Ao contrário das CO, as competições de CA caracterizam-se por provas mais longas com distâncias que normalmente variam entre 45 km e 1000 km, devendo ser percorridas no menor tempo possível, com duração de aproximadamente 6 horas a 10 dias. Além disso, ao analisarmos exigências específicas que fazem parte das competições de CA podemos observar, também, exigências fisiológicas de caráter predominantemente anaeróbico. Nestas situações, é necessária a execução de esforços físicos de grande intensidade e curta duração que exigem do organismo um rápido fornecimento de energia como travessias de cânions, escalada de morros íngremes, transposição de rios com fortes corredeiras e etc. Neste sentido, as CAs caracterizam-se por ser um esporte predominantemente aeróbico com importantes exigências anaeróbicas ao longo da prova (MANN; SCHAD, 2001). Somados aos aspectos fisiológicos encontra-se a necessidade de privação do sono nas competições com distâncias mais longas como um fator estratégico da prova.

Dentre os fatores técnicos e cognitivos que influenciam o desempenho das equipes de CA, podemos ressaltar a necessidade de homogeneidade do condicionamento físico entre os integrantes da equipe, visto que as regras do esporte exigem que a equipe atinja os PCs e a linha de chegada completa. Além disso, o desempenho da equipe na navegação é um fator essencial para o resultado final, pois a conclusão da prova de forma rápida e com menos erros torna a competição menos dispendiosa do ponto de vista bioenergético.

Dessa forma, como não há um consenso na literatura sobre os fatores determinantes do desempenho nesse esporte, o objetivo deste estudo foi correlacionar os valores relativos às equipes, às mulheres e aos navegadores nas variáveis idade, $\mathrm{VO}_{2 \text { máx’ }}, \mathrm{VO}_{2} 2^{\circ} \mathrm{LV}$, $\%$ 2'LV e tempo de teste correspondente ao segundo limar ventilatório (Temp_2LV) com o desempenho de suas equipes em competições de CA. 


\section{Material e Método}

\section{Sujeitos}

O presente trabalho realizado após um período de competições de CAé do tipo transversal. Sua amostra é composta pelas seis melhores equipes classificadas durante um campeonato de CA composto por quatro etapas realizadas ao longo de um ano, com distância média de $60 \mathrm{~km}$ por etapa, na categoria quarteto misto (três homens e uma mulher), totalizando 24 atletas, 18 do sexo masculino e seis do sexo feminino de nível competitivo regional e nacional (massa: 68,4 \pm 9,0 kg; estatura: 171,8 \pm 9,8 cm). As modalidades presentes no circuito foram navegação, corrida, Mountain Bike, Bóia-Cross, Rapel e Natação. Todos os participantes eram voluntários, foram esclarecidos previamente a respeito dos objetivos e procedimentos deste estudo e assinaram Termo de Consentimento Livre e Esclarecido aprovado pelo Comitê de Ética da Universidade Federal do Rio Grande do Sul, conforme a resolução 196/96 do Conselho Nacional de Saúde.

\section{Procedimento Experimental}

Todos os indivíduos foram submetidos a teste progressivo máximo em esteira com o

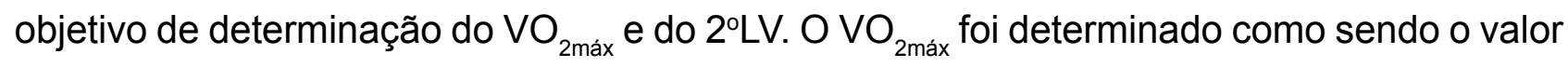
de consumo de oxigênio $\left(\mathrm{VO}_{2}\right)$ relativo mais alto encontrado entre as médias de intervalos de 30 s coletado continuamente utilizando circuito de espirometria aberta (Medical Graphics CPX/D, Arizona, EUA). A análise das curvas ventilatórias foi realizada por dois avaliadores experientes a partir do processamento dos dados "breath by breath" pela média de cinco dados a cada sete excluindo-se os valores mais altos e o mais baixo para $\mathrm{VO}_{2}$, produção de gás carbônico $\left(\mathrm{VCO}_{2}\right)$, ventilação minuto $(\mathrm{VE})$ e pressão expiratória de gás carbônico $\left(\mathrm{PECO}_{2}\right)$. Para determinação do $2^{\circ} \mathrm{LV}$ tomava-se como referência o ponto no qual se verificou um aumento exponencial da curva de ventilação minuto (VE), quando ocorresse um aumento do VE/VCO 2 ou quando ocorresse uma queda na PECO2. (CAIOZZO et al. 1982, AMANN et al. 2004). Estudos têm verificado que a utilização de métodos combinados para determinação dos limiares ventilatórios resulta em maior acuracidade (GASKILL et al. 2001). Antes de cada teste foram realizados os procedimentos básicos de calibração 
manual do sistema de espirometria através de uma seringa de três litros e do sistema de analise de gases por meio de uma mistura de gases de conteúdo conhecido de acordo com as normas do fabricante.

\section{Protocolos de Testes}

O protocolo utilizado caracterizou-se por aumentos progressivos da velocidade e da inclinação da esteira (IMBRAMED 10200ATL, Porto Alegre). O teste iniciava com velocidade a $5 \mathrm{~km}^{*} \mathrm{~h}^{-1}$ durante um min. e nível de inclinação da esteira de $1 \%$. Após isso, foram realizados aumentos progressivos na velocidade da esteira de $0,5 \mathrm{~km}^{*} \mathrm{~h}^{-1}$ a cada $30 \mathrm{~s}$ e, na inclinação, de $1 \%$ a cada $30 \mathrm{~s}$ a partir da velocidade de $12 \mathrm{~km}^{*} \mathrm{~h}^{-1}$. A velocidade ficava constante a partir de $17 \mathrm{~km}^{\star} \mathrm{h}^{-1}$. O término do teste era determinado por decisão voluntária do executante ou quando eram observados sintomas de limite máximo de esforço e incapacidade de manter a potência de trabalho. Os resultados deste teste foram considerados satisfatórios quando os indivíduos atingiam os seguintes critérios: valores de freqüência cardíaca máxima maior ou igual a $90 \%$ da freqüência cardíaca máxima prevista pela idade e taxa de troca respiratória maior do que 1.1 (TAYLOR et al. 1955). Esses testes foram realizados 15 a 20 dias após a última prova do campeonato de CA.

\section{Análise Estatística}

Os dados de $\mathrm{VO}_{2 \text { máx }}$ e das variáveis obtidas no $20 \mathrm{LV}\left(\mathrm{VO}_{2} 2^{\circ} \mathrm{LV}, \%\right.$ 2 $\left.2^{\circ} \mathrm{LV}, \mathrm{Temp} 2^{\circ} \mathrm{LV}\right)$ foram apresentados através da estatística descritiva (média e desvio-padrão). A normalidade e a homogeneidade dos dados foram avaliadas pelo teste de Shapiro-Wilk (Lilliefors) e Levene, respectivamente $(p>0,05)$. Para a comparação das médias entre as equipes foi utilizada a análise de variância (ANOVA One-way) e teste Post-Hoc (Tukey). Para verificar a correlação entre variáveis analisadas foi utilizada a Correlação Linear de Produto Momento de Pearson e a Correlação de Spearman (classificação das equipes na competição de CA). O nível de significância adotado foi $p<0,05$. O pacote estatístico utilizado foi o SPSS v.s. 14.0 for Windows. 


\section{Resultados}

A Tabela 1 apresenta as médias e os desvios padrões dos homens, mulheres, navegadores e do total dos participantes do estudo nas variáveis analisadas.

TABELA 1: Média e desvio-padrão $(\mathrm{dp})$ das variáveis $\mathrm{VO}_{2 \text { máx }}, \mathrm{VO}_{2} 2^{\circ} \mathrm{LV}, \% 2^{\circ} \mathrm{LV}$, Temp_2'LV e idade de todos os atletas (total), divididos por sexo e por função (navegadores).

\begin{tabular}{|c|c|c|c|c|}
\hline $\begin{array}{c}\mathrm{VO}_{2 \operatorname{máx}} \\
\left(\mathrm{ml}^{*} \mathrm{~kg}^{-1 *} \min ^{-1}\right)\end{array}$ & $\begin{array}{c}\mathrm{VO}_{2-} 2^{\circ} \mathrm{LV} \\
\left(\mathrm{ml}^{*} \mathrm{~kg}^{-1 *} \min ^{-1}\right)\end{array}$ & $\begin{array}{c}\% \_2{ }^{\circ} L V \\
(\%)\end{array}$ & $\begin{array}{c}\text { Temp_2 }{ }^{\circ} \mathrm{LV} \\
(\min )\end{array}$ & $\begin{array}{l}\text { Idade } \\
\text { (anos) }\end{array}$ \\
\hline média $\pm d p$ & média $\pm d p$ & média $\pm d p$ & média $\pm d p$ & média $\pm d p$ \\
\hline $51,8 \pm 5,9$ & $42,2 \pm 5,4$ & $81,4 \pm 5,6$ & $10: 47 \pm 1: 49$ & $28,0 \pm 5,7$ \\
\hline $53,0 \pm 6,2$ & $43,7 \pm 5,3$ & $82,5 \pm 5,3$ & $11: 21 \pm 1: 35$ & $27,4 \pm 5,4$ \\
\hline $47,3 \pm 2,4$ & $37,1 \pm 1,4$ & $79,9 \pm 2,4$ & $08: 30 \pm 0: 23$ & $29,8 \pm 6,9$ \\
\hline $51,7 \pm 4,1$ & $44,2 \pm 5,1$ & $84,8 \pm 5,7$ & $11: 30 \pm 1: 32$ & $31,14,0$ \\
\hline
\end{tabular}

A análise dos valores de $\mathrm{VO}_{2 \text { máx }}, \mathrm{VO}_{2} 2^{\circ} \mathrm{LV}, \%$ _ $2^{\circ} \mathrm{LV}, \mathrm{Temp} 2^{\circ} \mathrm{LV}$ e idade apresentaram normalidade e homogeneidade $(p>0,05)$. Ao comparar as equipes, verifica-se não haver diferenças estatisticamente significativas entre as médias das variáveis analisadas $(p>0,05)$ (TABELA 2). 
TABELA 2: Média e desvio-padrão (Média) e valores individuais de mulheres e

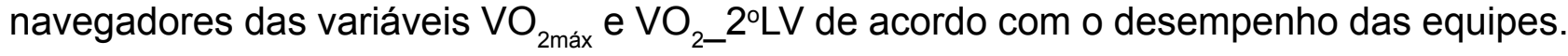

$\begin{array}{lcc}\text { Desempenho } & \mathrm{VO}_{2 \text { máx }} & \mathrm{VO}_{2}{ }^{2}{ }^{\circ} \mathrm{LV} \\ \text { da Equipe } & \left(\mathrm{ml}^{*} \mathrm{~kg}^{-1 *} \mathrm{~min}^{-1}\right) & \left(\mathrm{ml}^{*} \mathrm{~kg}^{-1 *} \mathrm{~min}^{-1}\right)\end{array}$

Média $\left({ }^{*}\right)$ Mulher Navegador Média $\left(^{*}\right)$ Mulher Navegador

\begin{tabular}{|c|c|c|c|c|c|c|}
\hline $1^{\circ}$ lugar & $57,9 \pm 5,2$ & 51,5 & 57,3 & $44,9 \pm 6,7$ & 35,0 & 49,4 \\
\hline $2^{\circ}$ lugar & $48,3 \pm 3,5$ & 45,4 & 45,2 & $39,8 \pm 3,2$ & 37,0 & 38,7 \\
\hline $3^{\circ}$ lugar & $55,8 \pm 3,3$ & 45,0 & 53,5 & $47,3 \pm 1,6$ & 37,1 & 46,2 \\
\hline $4^{\circ}$ lugar & $49,4 \pm 5,0$ & 46,2 & 53,9 & $43,5 \pm 5,9$ & 38,0 & 50,5 \\
\hline $5^{\circ}$ lugar & $52,9 \pm 7,2$ & 48,5 & 49,5 & $42,5 \pm 6,6$ & 39,3 & 38,2 \\
\hline $6^{\circ}$ lugar & $48,7 \pm 5,0$ & 47,7 & 50,4 & $37,6 \pm 3,4$ & 36,4 & 40,1 \\
\hline
\end{tabular}

Baixas correlações foram encontradas entre a média dos parâmetros fisiológicos de cada equipe, bem como os valores individuais de mulheres e de navegadores, com o desempenho na competição (TABELA 3). Por outro lado, boas correlações foram encontradas entre a idade das mulheres e dos navegadores com o desempenho de suas equipes (FIGURA 1).

TABELA 3: Correlação entre o desempenho das equipes com os valores das variáveis $\mathrm{VO}_{2 \text { máx }}, \mathrm{VO}_{2} 2^{\circ} \mathrm{LV}, \% \_2^{\circ} \mathrm{LV}$, Temp_2 $2^{\circ} \mathrm{LV}$ e idade das mulheres, dos navegadores e da média de cada equipe.

\begin{tabular}{|c|c|c|c|c|c|}
\hline & $\begin{array}{c}\mathrm{VO}_{2 \operatorname{máx}} \\
\left(\mathrm{ml}^{*} \mathrm{~kg}^{-1 *} \min ^{-1}\right)\end{array}$ & $\begin{array}{c}\mathrm{VO}_{2} 2^{\circ} \mathrm{LV} \\
\left(\mathrm{ml}^{*} \mathrm{~kg}^{-1 *} \mathrm{~min}^{-1}\right)\end{array}$ & $\begin{array}{c}\% 2^{\circ} \mathrm{LV} \\
(\%)\end{array}$ & $\begin{array}{c}\text { Temp_2 }{ }^{\circ} \mathrm{LV} \\
(\min )\end{array}$ & $\begin{array}{l}\text { Idade } \\
\text { (anos) }\end{array}$ \\
\hline Mulheres & 0,03 & 0,43 & $-0,30$ & 0,22 & $-0,90^{*}$ \\
\hline Navegadores & $-0,31$ & $-0,31$ & $-0,43$ & $-0,52$ & $-0,77$ \\
\hline Média Equipe & $-0,34$ & $-0,22$ & $-0,14$ & $-0,18$ & $-0,32$ \\
\hline
\end{tabular}



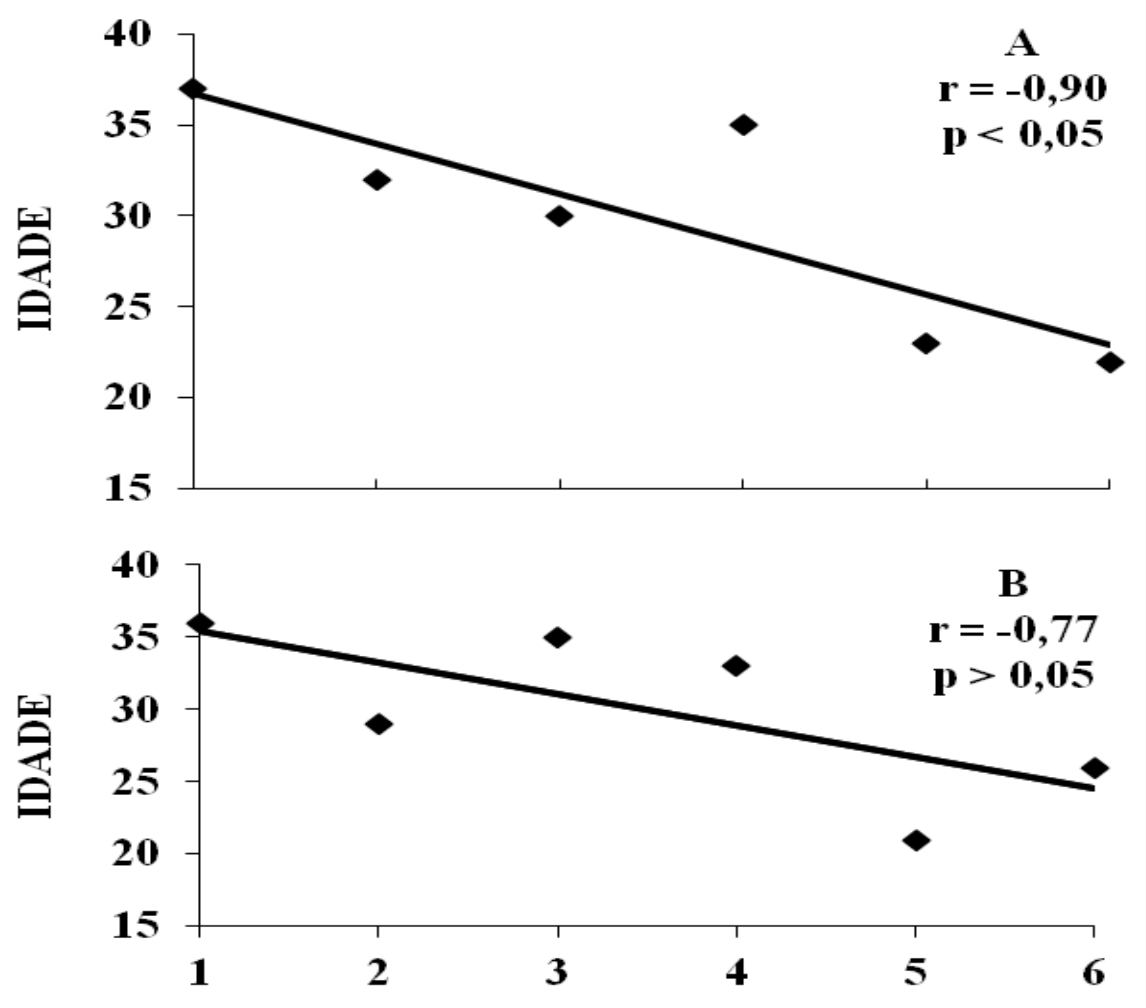

DESEMPENHO

FIGURA 1: Correlação entre a idade (anos) das mulheres $(A)$ e dos navegadores $(B)$ com o desempenho (classificação) de suas equipes na competição.

\section{Discussão}

Os valores das médias de $\mathrm{VO}_{2 \text { máx }}$ nos homens foram bastante inferiores às apresentadas em trabalhos com corredores de cross-country 72,1 $\mathrm{ml}^{*} \mathrm{~kg}^{-1 *} \mathrm{~min}^{-1}$ (BULBULIAN et al. 1986), maratonistas $65,7 \mathrm{ml}^{*} \mathrm{~kg}^{-1 *} \mathrm{~min}^{-1}$ (PÉRONNET et al. 1987), atletas amadores de triatlo $62 \mathrm{ml}^{*} \mathrm{~kg}^{-1 *} \mathrm{~min}^{-1}$ (BUTTS et al. 1991), triatletas especialistas em "sprint triatlo" $63,7 \mathrm{ml}^{*} \mathrm{~kg}^{-1 *} \mathrm{~min}^{-1}$ (SLEIVERT; WENGER, 1993) e atletas de CO 61,6 $\mathrm{ml}^{*} \mathrm{~kg}^{-1 *} \mathrm{~min}^{-1}$ (KNOWLTON et al. 1980) e $74,3 \mathrm{ml}^{*} \mathrm{~kg}^{-1 *} \mathrm{~min}^{-1}$ (JENSEN et al. 1994). Nas mulheres, essa variável foi um pouco inferior às médias apresentadas por atletas femininas de outros esportes de longa duração como triatlo $50,7 \mathrm{ml}^{*} \mathrm{~kg}^{-1 *} \mathrm{~min}^{-1}$ (BUTTS et al. 1991), 51,4 $\mathrm{ml}^{*} \mathrm{~kg}^{-1 *} \mathrm{~min}^{-1}$ (SLEIVERT; WENGER, 1993) e atletas de CO 46,1 $\mathrm{ml}^{*} \mathrm{~kg}^{-1 *} \mathrm{~min}^{-1}$ (KNOWLTON et al. 1980), $59 \mathrm{ml}^{*} \mathrm{~kg}^{-1 *} \min ^{-1}$ (JENSEN et al. 1994).

Podemos atribuir esses resultados, em primeiro lugar, ao fato dos valores de $\mathrm{VO}_{2 \text { máx }}$ e o $\mathrm{VO}_{2} 2^{\circ} \mathrm{LV}$ estarem sendo comparados com esportes que apresentam características 
bastante diferentes da CA. Na CA a noção de multifatoriedade está bastante evidente, pois além da exigência de desempenho físico em modalidades esportivas diferentes, a irregularidade da prova em relação ao terreno e à sua duração exige dos atletas uma preparação diferenciada, composta pelo desenvolvimento de diversos tipos de habilidades técnicas, diminuindo o volume do treinamento para uma modalidade específica, ao contrário do que acontece com corredores, ciclistas, triatletas e atletas de corrida de orientação. Soma-se a isso o fato dos atletas de CA necessitar a utilização de estratégias diferenciadas para melhorar seu desempenho durante as competições, tais como a privação de sono e do descanso, além de precisar suportar condições ambientais adversas. Outra possibilidade é o fato da CA ser um esporte em desenvolvimento no Brasil, apesar de estar obtendo resultados expressivos em nível internacional.

A semelhança entre os valores médios das equipes é interessante (TABELA 2), pois ressalta o atual nível competitivo entre as equipes participantes do esporte. Já a semelhança entre as variâncias dos fatores preditores de performance, verificado através do teste de homogeneidade, revela que as diferenças existentes entre os componentes de uma mesma equipe se assemelha com a variabilidade encontrada em outras equipes. Esse dado é interessante, visto que, para o bom desempenho de uma equipe é importante não apenas a existência de médias elevadas, mas, também, baixa variabilidade e regularidade no condicionamento físico dentro do grupo, devido à necessidade dos atletas de uma mesma equipe alcançar os PCs ao mesmo tempo.

De uma forma geral, o $\mathrm{VO}_{2 \text { máx }}$ e o $2^{\circ} \mathrm{LV}$ têm sido apontados como os principais marcadores fisiológicos de desempenho em esportes de longa distância (NOAKES, 1988; CREAGH; REILLY, 1997; BASSETT; HOWLEY, 2000; NOAKES, 2000). Em estudos anteriores, foram encontradas boas correlações entre VO2máx e desempenho em corredores universitários $(r=-0,76)$ (TANAKA et al. 1984) e atletas de triatlo masculinos ( $r=-0,84)$ (BUTTS et al. 1991) e femininos ( $r=-0,88)$ (SLEIVERT; WENGER, 1993). Entretanto, os resultados encontrados no presente estudo apontaram para uma baixa correlação entre $\circ \mathrm{VO}_{2 \text { máx }}$ médio de cada equipe com o seu desempenho na competição $(r=-0,34, p>0,05)$ (TABELA 3). Resultados semelhantes foram encontrados também em estudos com triatletas da elite que competiam em provas de longa distância como o meio ironman e o próprio ironman (De 
VITO et al. 1995; O'TOOLE; DOUGLAS, 1995), assim como em experientes corredores de 10 km (CONLEY; KRAHENBUHL, 1980), em corredores de cross-country (BULBULIAN et al. 1986) e em maratonistas (PÉRONNET et al. 1987).

Apesar da identificação inicial do condicionamento aeróbico como característica predominante durante uma prova de CA (MANN; SCHAD, 2001), a baixa correlação entre o $\mathrm{VO}_{2 \text { máx }}$ e o desempenho das equipes demonstra que, apesar de ser uma qualidade imprescindível, por si só não define o resultado final da competição. Entretanto, vale ressaltar que mesmo os estudos que não encontraram boas correlações do $\mathrm{VO}_{2 \text { máx }}$ com os resultados em competições, observaram que todos os atletas analisados possuíam altos $\mathrm{VO}_{2 \text { máx }}$. Conclui-se, então, que é uma característica extremamente importante a ser treinada quando se objetiva o alto desempenho em provas de longa distância, inclusive para promover mais rapidamente a recuperação de parâmetros anaeróbicos durante a competição, como é o caso das CA.

$\mathrm{O} \mathrm{VO}_{2 \text { máx }}$ se mostrou limitado como preditor de desempenho a medida que os grupos de atletas observados são homogêneos quanto a este fator, o que determina a utilização de outras características fisiológicas para diferenciar estes atletas. Tal fato tem sido há muito tempo observado em pesquisas com experientes corredores de $10 \mathrm{~km}$ (CONLEY; KRAHENBUHL, 1980), corredores de cross-country (BULBULIAN et al. 1986), maratonistas (PÉRONNET et al. 1987) e triatletas (KOHRT et al. 1987; SLEIVERT; ROWLANDS, 1996). Percebe-se então, que, enquanto um alto $\mathrm{VO}_{2 \text { máx }}$ pode ser um pré-requisito para o sucesso em corridas de longa distância, outras variáveis fisiológicas, também, contribuem muito para o atual desempenho no esporte de alto rendimento (CONLEY; KRAHENBUHL, 1980).

O 2ㄴV, também, tem sido reportado como um importante contribuidor para o desempenho em provas de longa distância. A maior parte dos estudos tem encontrado boas correlações com desempenho ao analisarem o $\mathrm{VO}_{2} 2^{\circ} \mathrm{LV}$ de triatletas $(r=-0,88)$ (TANAKA et al. 1984), o \%_2 ${ }^{\circ} L V$ de maratonistas $(r=-0,85)$ (PÉRONNET et al. 1987) e a Vel_2 ${ }^{\circ} L V$ tanto de corredores $(r=-0,96)$ (MAFFULLI et al. 1991) e $(r=-0,89)$ (SLEIVERT; WENGER, 1993) como de triatletas $(r=-0,81)$ (TANAKA et al. 1984). Isto se deve, principalmente, ao retardo do metabolismo anaeróbio durante o aumento da intensidade de trabalho (PÉRONNET et al. 1987). Esta característica se mostra relevante, quando os atletas competem em provas 
onde a mudança de intensidade é constante, como é o caso de corredores de cross-country (BULBULIAN et al. 1986). No caso das CA, a importância de um alto $2^{\circ}$ LV se fará presente, também, em momentos onde os terrenos com grandes desníveis e obstáculos naturais exigirão dos atletas executarem curtas explosões de grande intensidade que necessitem de um rápido acesso a um grande volume de energia ou, então, evitar a diminuição do ritmo dos atletas durante a prova (MANN; SCHAAD, 2001). Esta afirmação corrobora, a princípio, com a importância de um alto 2oLV no desempenho final das equipes nestas competições. No entanto, os resultados deste estudo apontaram, também, para uma baixa correlação do $\mathrm{VO}_{2} 2^{\circ} \mathrm{LV}(r=-0,22)$ do $\% \_2^{\circ} \mathrm{LV}(r=-0,14)$ e do Temp_2 $2^{\circ} \mathrm{LV}(r=-0,18)$ dos atletas de CA com o seu desempenho nas competições ( $p>0,05)$ (TABELA 3).

Isso significa que, contrariando diversos estudos, a importância do $2^{\circ} \mathrm{LV}$ como variável preditora de desempenho parece ter sido minimizada na CA. Esse aspecto reforça, ainda mais, as peculiaridades deste esporte, caracterizado pela necessidade de execução de diferentes modalidades esportivas em um ambiente adverso e não constante, as quais exigem de todos os componentes da equipe, não apenas um aprimoramento fisiológico, mas uma preparação ampla e multifatorial para poderem alcançar o sucesso na realização dessas provas. Um estudo realizado em atletas de alto nível de mountain bike verificou que o condicionamento aeróbico verificado através do $\mathrm{VO}_{2} 2^{\circ} \mathrm{LV}$, bem como, a potência produzida nesta fase, responderam cerca de $40 \%$ do desempenho dos atletas, destacando os autores a importância de outros fatores, dentre eles, aspectos técnicos para o desempenho (IMPELLIZZERI et al. 2005). Neste sentido, outros elementos podem estar contribuindo, também, para o desempenho final das equipes de CA (ANGLEM et al. 2008; LUCAS et al. 2009; LEVADA-PIRES et al. 2010).

Outra questão que poderia estar influenciando no desempenho das equipes de CA seria a probabilidade do ritmo da equipe ser determinada pelo condicionamento físico da integrante feminina. Sabe-se que o condicionamento físico das mulheres tende a ser menor do que o dos homens. Dentre os diversos fatores, podemos citar diferenças na estrutura óssea implicando em passadas menores, maiores percentuais de tecido adiposo estabelecendo uma proporção desfavorável entre a massa muscular ativa e massa corporal total, menor volume cardíaco, menor volume sangüíneo, menor quantidade de hemoglobinas, menor 
eficiência respiratória, etc (WILMORE et al. 2010). Entretanto, do mesmo modo que foram encontradas com os valores de todos os atletas, baixas correlações foram verificadas entre o $\mathrm{VO}_{2 \text { máx }}$ e as variáveis obtidas no $2^{\circ} \mathrm{LV}$ das atletas femininas com o desempenho de suas equipes $\left(\mathrm{VO}_{2 \text { máx }}: r=0,03 ; V_{2} 2^{\circ} \mathrm{LV}: r=0,43 ; \%\right.$ 2 ${ }^{\circ} \mathrm{LV}: \mathrm{r}=-0,30$ e Temp_2 $L V$ : $r=-0,22)(p>0,05)($ TABELA 3). Neste sentido, outros fatores parecem estar minimizando a importância das variáveis fisiológicas na predição do desempenho de CA, tornando o estudo deste esporte ainda mais complexo.

Dentre outros elementos que podem influenciar no resultado final das equipes de CA podemos citar o desempenho do navegador. Dos quatro atletas que compõe uma equipe de CA o navegador é o integrante com maior responsabilidade. É o único com função preestabelecida, sendo o responsável por guiar a equipe pelo melhor trajeto da largada até a chegada. Sendo assim, é conveniente afirmar que a equipe é totalmente dependente do desempenho técnico, psicológico e também fisiológico deste atleta. O navegador, além da preparação física para suportar as longas distâncias percorridas, necessita de preparação técnica específica para navegação e psicológica para saber administrar os momentos de conflito e tensão inerentes à tomada de decisão.

Durante as competições estes fatores podem exercer influências entre si, ou seja, se algum desses fatores estiver abaixo do nível ideal, pode influenciar negativamente também os outros aspectos. A inter-relação entre o fator fisiológico e o psicológico foi sugerida em trabalho realizado com pilotos de helicóptero do exército brasileiro, revelando que um elevado padrão de desempenho físico aeróbico pode exercer um efeito positivo no comportamento psicofisiológico dos pilotos e que a manutenção deste padrão em patamares superiores poderá vir a aperfeiçoar suas performances na atividade aérea (RIBAS; RIBEIRO, 2003). Em outro estudo realizado com militares, os indivíduos mais bem condicionados aerobicamente tiveram melhor capacidade de relaxamento frente a um estímulo estressor (DUARTE et al. 2003). Em outro trabalho, grupos menos condicionados fisicamente tiveram uma maior reatividade cardíaca e um maior desgaste metabólico em resposta a um estímulo estressor (BOUTCHER et al. 2001). Ando et al. (2005) verificaram que o aumento do tempo de reação (TR) do campo visual periférico durante exercício de alta intensidade foi inversamente proporcional ao $\mathrm{VO}_{2 \text { máx }}$, demonstrando que a alta capacidade aeróbia atenuaria o aumento 
do TR durante o exercício exaustivo. Dessa forma, os níveis de estresse e a percepção espacial do indivíduo poderiam ser influenciados por sua capacidade aeróbia, o que poderia influenciar na tomada de decisão e, conseqüentemente, no desempenho competitivo.

Apesar de não ser um consenso (MOYNA et al. 1999; SPALDING et al. 2000), parte da literatura vem demonstrando que é importante possuir um bom condicionamento aeróbico, para minimizar a perda de desempenho de tomada de decisão em momentos de estresse. Este aspecto pode ser benéfico, principalmente, para os navegadores das equipes de CA, que são exigidos cognitivamente durante a navegação em diversos momentos das competições. No entanto, em nosso trabalho foram encontradas, também, baixas correlações entre $0 \mathrm{VO}_{2 \text { máx }}$ e as variáveis obtidas no $2^{\circ} \mathrm{LV}$ dos navegadores com o desempenho de suas equipes na competição $\left(\mathrm{VO}_{2 \text { máx }}: r=-0,31 ; \mathrm{VO}_{2} 2^{\circ} \mathrm{LV}: r=-0,31\right.$; \%_2 ${ }^{\circ} L V: r=-0,43 ;$ Temp_2 $\left.{ }^{\circ} L V: r=-0,52\right)(p>0,05)$ (TABELA 3). Embora esses resultados não tenham apontado uma relação direta entre a capacidade fisiológica do navegador e o desempenho de sua equipe é importante que tenhamos atenção especial para outras características peculiares a ele.

Um aspecto analisado e que de mostrou boa correlação foi a relação entre idade dos navegadores com a ordem de classificação das equipes em uma competição de CA $(r=$ $-0,77)(p>0,05)$ (FIGURA 1). Este resultado parece revelar a existência de relação entre as experiências competitivas e de orientação dos navegadores de forma a influenciar positivamente o desempenho de suas equipes. Navegadores mais experientes em competições sofreriam menos efeito do estresse sobre a tomada de decisão, repercutindo na menor quantidade de decisões erradas. Em trabalho realizado com atletas de $\mathrm{CO}$, defendeu-se que os indivíduos com maior experiência competitiva teriam maior capacidade de observar possíveis erros na carta topográfica ou obstáculos no terreno no momento em que eles aparecessem e assim contorná-los mais facilmente (DIAS; DANTAS, 2004). Estes resultados não significam, necessariamente, que navegadores mais velhos teriam melhores condições técnicas e cognitivas do que navegadores mais jovens, mas, possivelmente, estaria sendo um fator de grande contribuição para alcançarem bons resultados nas competições, através de uma navegação mais tranqüila e com menor quantidade de erros.

Somando-se a esse aspecto, está o fato de ter-se encontrado uma significativa 
correlação entre a idade da integrante feminina com a ordem de classificação das equipes na competição de CA $(r=-0,90, p<0,05)$ (FIGURA 1). Independente do condicionamento físico, a mulher mais velha, também, estaria contribuindo com o aumento do desempenho da equipe, possivelmente, por estar mais adaptada a suportar as condições adversas das competições e tecnicamente preparada para a transposição dos obstáculos. Entretanto, é importante ressaltar que esses resultados não significam que as equipes constituídas de indivíduos mais velhos tenham mais sucesso na CA, pois foram encontradas fracas correlações ao analisar a idade de todos os atletas $(r=-0,31)$, de todos os indivíduos do sexo masculino $(r=-0,11)$ e o valor médio de idade das equipes $(r=-0,43)$ com o desempenho $(p>0,05)$. Analisando-se deste ponto de vista, no entanto, navegadores e mulheres mais velhas parecem favorecer resultados positivos em competições, ressaltando, dessa forma, o caráter multifatorial existente na CA. Apesar da idade mais elevada não representar, necessariamente, maior maturidade e uma melhor preparação técnica ou experiência, esses resultados reforçam a importância que a navegação e a necessidade de superação de adversidades possuem dentro da competição, podendo ser considerados fatores relevantes em relação à predição do desempenho em CA.

\section{Conclusão}

De uma forma geral, o conceito de multifatoriedade mostrou-se bastante relevante no presente trabalho, destacando que nenhum dos parâmetros fisiológicos analisados mostrou boa correlação com o desempenho de equipes de CA em competição. Nas CA este conceito parece ter maior relevância visto que é necessário o conhecimento de diversas modalidades esportivas, destacando-se as técnicas de orientação. Tal característica técnica e cognitiva parece estar se sobressaindo sobre o aspecto físico nas competições, porém, isto não significa que o fator fisiológico não seja importante. Mesmo havendo baixas correlações entre os valores de $\mathrm{VO}_{2 \text { máx }}$ e das variáveis obtidas no $2^{\circ} \mathrm{LV}$ com o desempenho, a equipe campeã apresentou os melhores resultados fisiológicos entre as equipes analisadas. Esta análise reforça o fato de que, mesmo sendo difícil a predição do desempenho através dos valores de $\mathrm{VO}_{2 \operatorname{máx}}$ e $2^{\circ} \mathrm{LV}$ dos atletas, uma adequada preparação fisiológica é um importante pré-requisito para se almejar o alto desempenho. É aconselhável, dessa forma, que as 
equipes devam estar muito bem preparadas em todos os aspectos, a fim de superarem com o mínimo de esforço físico e cognitivo os desafios peculiares a cada competição, que podem em alguns momentos exigir mais da navegação e em outros momentos exigir mais do condicionamento físico. Para futuras pesquisas, sugere-se que sejam analisadas não somente fatores fisiológicos, mas, também, fatores técnicos e psicológicos relacionados ao desempenho da navegação, podendo assim, estabelecer percentuais de contribuição de cada fator no desempenho em competições de CA.

\section{Referências}

ACKERMAN, K. J.; KNOWLTON, R. G.; SEDLOCK, D. H. Physiological stress and performance changes in response to beginning level orienteering. J. Sports Med. Phys. Fitness. v. 25, n. 3, p. 175-182, 1985.

AINSLIE, P. N. et al. Physiological and metabolic responses to a hill walk. J. Appl. Physiol. v. 92, n. 1, p. 179-187, 2002.

AMANN, M.; SUBUDHI, A.; FOSTER, C. Influence of testing protocol on ventilatory thresholds and cycling performance. Med. Sci. Sports Exerc. v. 36, n. 4, p. 613-622, 2004.

ANDO, S. et al. Increase in reaction time for the peripheral visual field during exercise above the ventilatory threshold. Eur. J. Appl. Physiol. v. 94, n. 4, p. 461-467, 2005.

ANGLEM, N. et al. Mood, illness and injury responses and recovery with adventure racing. Wilderness Environ Med. v. 19, n. 1, p. 30-38, 2008.

BASSETT, D. R.; HOWLEY, E. T. Limiting factors for maximum oxygen uptake and determinants of endurance performance. Med. Sci. Sports Exerc. v. 32, n. 1, p. 70-84, 2000.

BOUTCHER, S. H.; NURHAYATI, Y.; MCLAREN, P. F. Cardiovascular response of trained and untrained old men to mental challenge. Med. Sci. Sports Exerc. v. 33, n. 4, p. 659-664, 2001.

BULBULIAN, R.; WILCOX, A. R.; DARABOS, B. L. Anaerobic contribution to distance running performance of trained cross-country athletes. Med. Sci. Sports Exerc. v. 18, n. 1, p. 107-113, 1986.

BUTTS, N. K.; HENRY, B. A.; MCLEAN, D. Correlations between VO ${ }_{2 \text { máx }}$ and performance 
times of recreational triathletes. J. Sports Med. Phys. Fitness. v. 31, n. 3, p. 399-44, 1991.

CAIOZZO, V. J. et al. A comparison of gas exchange indices used to detect the anaerobic threshold. J. Appl Physiol. v. 53, n. 5, p. 1184-1189, 1982.

CAVANAGH, P. R.; KRAM, R. The efficiency of human movement: a statement of the problem. Med. Sci. Sports Exerc. v. 17, n. 3, p. 304-308, 1985.

CONLEY, D. L.; KRAHENBUHL, G. K. Running economy and distance running performance of highly trained athletes. Med. Sci. Sports Exerc. v. 12, n. 5, p. 357-360, 1980.

CREAGH, U.; REILLY, T. Physiological and biomechanical aspects of orienteering. Sports Med. v. 24, n. 6, p. 409-416, 1997.

DANIELS, J. T. A physiologist's view of running economy. Med. Sci. Sports Exerc. v. 17, n. 3, p. 332-338, 1985.

De VITO, G. et al. Decrease of endurance performance during olympic triathlon. Int. J. Sports Med. v. 16, n. 1, p. 24-28, 1995.

DIAS, A. C.; DANTAS, E. H. M. A tomada de decisão na orientação. Rev. Edu. Física. v. 128, p. $12-17,2004$.

DICKHUTH, H. H. et al. Ventilatory, lactate-derived and catecholamine thresholds during incremental treadmill running: relationship and reproducibility. Int. J. Sports Med. v. 20, n. 2, p. 122-127 1999.

DRESEL, U. Lactate acidosis with different stages in the course of a competitive orienteering performance. Sci. J. Orienteering. v.1, p. 4-13, 1985.

DUARTE, A. F. et al. Condicionamento físico aeróbio e reações psicofisiológicas a um teste de estresse. Rev. Edu. Física. v. 127, p. 4-9, 2003.

GASKILL, S. E. et al. Validity and reliability of combining three methods to determine ventilatory threshold. Med. Sci. Sports Exerc. v. 33, n. 11, p. 1841-1848, 2001.

IMPELLIZZERI, F. M. etal. Correlations between physiological variables and performance in high level cross country off road cyclists. Br J Sports Med. v. 39, n. 10, p.747-51, 2005.

JENSEN, K.; FRANCH, J.; KÄRKKÄINEN, O. Field measurements of oxygen uptake in elite orienteers during cross-country running using telemetry. Scand. J. Med. Sci. Sports. v. 4, p. 234-8, 1994. 
KNOWLTON, R. G. et al. Physiological and performance characteristics of United States championship class orienteers. Med. Sci. Sports Exerc. v. 12, n. 3, p. 164-9, 1980.

KOHRT, W. M. et al. Physiological responses of triathletes to maximal swimming, cycling, and running. Med. Sci. Sports Exerc. v. 19, n. 1, p. 51-55, 1987.

LEVADA-PIRES, A. C. et al. The effect of an adventure race on lymphocyte and neutrophil death. Eur J Appl Physiol. v. 109, n. 3, p. 447-53, 2010.

LUCAS, S. J. et al. The impact of 100 hours of exercise and sleep deprivation on cognitive function and physical capacities. J Sports Sci. v. 27, n. 7, p. 719-28, 2009.

MAFFULLI, N.; CAPASSO, G.; LANCIA, A. Anaerobic threshold and performance in middle and long distance running. J. Sports Med. Phys. Fitness. v. 31, n. 3, p. 332-338, 1991.

MANN, D.; SCHAAD, K. The complete guide to adventure racing. Long Island City, New York: Hatherleigh Press, 2001.

MARTIN, P. E.; MORGAN, D. W. Biomechanical considerations for economical walking and running. Med. Sci. Sports Exerc. v. 24, n. 4, p. 467-474, 1992.

MOYNA, N. M. et al. Relation between aerobic fitness level and stress induced alterations in neuroendocrine and immune function. Int. J. Sports Med. v. 20, n. 2, p. 136-141, 1999.

NOAKES, T. D. Implications of exercise testing for prediction of athletic performance: a contemporary perspective. Med. Sci. Sports Exerc. v. 20, n. 4, p. 319-330, 1988.

NOAKES, T. D. Physiological models to understand exercise fatigue and the adaptations that predict or enhance athletic performance. Scand. J. Med. Sci. Sports. v. 10, n. 3, p. 123-145, 2000.

O'TOOLE, M. L.; DOUGLAS, P. S. Applied physiology of triathlon. Sports Med. v. 19, n. 4, p. 251-267, 1995.

PÉRONNET, F. et al. Correlation between ventilatory threshold and endurance capability in marathon runners. Med. Sci. Sports Exerc. v. 19, n. 6, p. 610-615, 1987.

RIBAS, P. R.; RIBEIRO, L. C. S. Aptidão física e o controle do comportamento psicofisiológico de pilotos de helicópteros do exército brasileiro pelo biofeedback. Rev. Edu. Física. v. 127, p. 41-47, 2003.

SLEIVERT, G. G.; ROWLANDS, D. S. Physical and physiological factors associated 
with success in the triathlon. Sports Med. v. 22, n. 1, p. 8-18, 1996.

SLEIVERT, G. G.; WENGER, H. A. Physiological predictors of short-course triathlon performance. Med. Sci. Sports Exerc. v. 25, n. 7, p. 871-876, 1993.

SMEKAL, G. et al. Respiratory gas exchange and lactate measures during competitive orienteering. Med. Sci. Sports Exerc. v. 35, n. 4, p. 682-689, 2003.

SPALDING, T. W. et al. Vagal and cardiac reactivity to psychological stressors in trained and untrained men. Med. Sci. Sports Exerc. v. 32, n. 3, p. 581-591, 2000.

TANAKA, K. et al. A longitudinal assessment of anaerobic threshold and distance-running performance. Med. Sci. Sports Exerc. v. 16, n. 3, p. 278-282, 1984.

TAYLOR, H. L.; BUSKIRK, E.; HENSCHEL, A. Maximal oxygen intake as an objective measure of cardiorespiratory performance. J. Appl. Physiol. v. 8, n. 1, p. 73-80, 1955.

WILMORE, J. H; COSTILL, D. L; KENNEY, W. L. Fisiologia do Esporte e do Exercício. São Paulo: Manole, 2010. 


\section{RELATION BETWEEN PHYSICAL CONDITIONING AND AGE ON PERFORMANCE OF TEAMS OF ADVENTURE RACE}

ABSTRACT: The objective of this study was to correlate values of maximal oxygen consumption (VO2max) and evaluated variables in the second ventilatory threshold (VT2) of Adventure Race team. Six teams performed progressive test on treadmill to determine VO2max and VT2. There were not significant differences between the averages of the teams and the correlation with performance was low. Good coefficients were found between the age and performance for navigators $(r=-0.77)$ and women $(r=-0.90 ; p<0.05)$. Despite of the appropriate physical conditioning for the sport, the experience of the navigators and the women can have contributed to the performance of the teams

Keywords: Physical fitness. Oxygen consumption. Sports.

\section{RELACIÓN ENTRE LA CONDICIÓN FÍSICA Y LA EDAD EN EL RENDIMIENTO DE LOS EQUIPOS DE CARRERAS DE AVENTURA}

RESUMEN: El objetivo de este estudio fue correlacionar los valores de consumo máximo de oxígeno (VO2máx) y las variables evaluadas en el segundo umbral ventilatorio (UV2) en equipos de Carreras de Aventura. Seis equipos realizó prueba de esfuerzo progresivo para determinar el VO2máx y UV2. No se encontraron diferencias significativas entre las medias de los equipos y su correlación con el rendimiento era bajo. No se encontraron buenos coeficientes entre la edad y los navegadores de rendimiento $(r=-0,77)$ y mujeres $(r=-0,90$, $p<0,05)$. A pesar de la exigencia es la aptitud adecuada para el deporte, la experiencia de los navegadores y la mujer puede haber contribuido a la actuación de los equipos.

Palabras Clave: Acondicionamiento Físico. Consumo de Oxígeno. Deportes. 